\title{
QUELQUES PARTICULARITÉS NUTRITIONNELLES ET PHYSIOLOGIQUES DU POUSSIN NAIN $d w$
}

\author{
J. GUILLAUME \\ avec la collaboration technique de Michèle SAUveur, Joëlle Bruk̀re et Liliane Dupuy \\ Station de Recherches avicoles, \\ Centre de Recherches de Tours, I. N. R. A., \\ 37 - Nouzilly
}

\section{RÉSUMÉ}

Nous effectuons 3 expériences de courte durée en vue de rechercher l'influence du gène de nanisme récessif lié au sexe $d w$ sur le besoin azoté du poussin en croissance. Les animaux, élevés en cages individuelles, sont issus d'un croisement expérimental $D w d w \times \mathrm{d} w$ et comprennent par conséquent $50 \mathrm{p}$. I oo de nains et de normaux dans chaque sexe. Ils sont sexés à la naissance mais les phénotypes sont déterminés à $\mathbf{2} 2$ semaines après la fin des expériences proprement dites.

Dans l'essai $n^{0} \mathrm{I}$ les poussins sont nourris selon la méthode de l' " alimentation séparée ": ils reçoivent chaque jour une ration protéique pesée dans une mangeoire et disposent en permanence d'aliment protéiprive dans une autre mangeoire. On constate que mâles et femelles ont un comportement alimentaire différent, les mâles seuls équilibrant les protéines et l'énergie dans l'aliment total reconstitué. Dans tous les cas, les nains reconstituent un aliment plus riche en protéines ; leur taux de conversion est meilleur mais leur coefficient d'efficacité protéique est plus faible.

Dans les essais 2 et 3 , tous les animaux sont rationnés. Dans l'expérience 2, la moitié reçoit un régime $A$ marginal en protéines, l'autre moitié en régime $B$ excédentaire en protéines. Dans l'expérience 3 , le régime $B$ est remplacé par le régime $\mathrm{C}$ de même taux azoté que A mais plus énergétique. Dans les 2 essais, l'effet du gène est peu marqué et aucune interaction du I er ordre génotype $\times$ régime n'apparaît. Chez les femelles, on note cependant que le gain de poids est amélioré par le taux azoté de façon significative chez les naines seulement (essai I) et par le taux énergétique de façon plus marquée chez les normales (interaction significative - essai 2).

Les poussins nains semblent donc nécessiter un régime à rapport Calories/Protéines moins élevé mais d'autres essais sont nécessaires pour le vérifier. Le gène de nanisme semble influencer aussi le comportement alimentaire du jeune poussin.

Nous avons également effectué des mesures biochimiques et physiologiques sur des paires de frères ou de sœurs (l'un nain, l'autre normal) à I 2 semaines. Les sujets $d w$ ont des teneurs sanguines plus faibles en glucose, lipides totaux et cholestérol total. La concentration du muscle en acides aminés libres totaux est plus faible chez les nains et certains acides aminés (thréonine, sérine et glycine) sont spécialement diminués. Ces modifications sont très différentes de celles qu'induit le thiouracile.

Le thiouracile a un effet plus dépressif chez les femelles $d w$. L'étude du relâchement de ${ }^{131}$ I par la thyroïde montre que cette glande est moins active chez les nains ; la différence est cependant faible comparée à celle qui existe d'une famille à l'autre. 
On pense donc que la diminution de l'activité thyroïdienne n'est qu'un épiphénomène et que l'action première du gène réside soit dans la sécrétion ou la libération d'hormone de croissance soit dans la sensibilité des tissus à cette hormone.

\section{INTRODUCTION}

Le poussin porteur du gène $d w$ a fait 1'objet d'études génétiques (HuTT, I953; HutT, I969 ; MÉRAT, I969 ; JAAP et MOHAMmadian, I969 ; RiCARD, I970) et physiologiques (VAN Tienhoven et al., I966; Mérat et GuIllaume, I969; Rajaratnam, SelvarajaH et Summers, I969; Mohammadian, I969). On sait encore peu de choses sur ses besoins nutritionnels durant la croissance. Par rapport aux animaux normaux, BERNIER et ARSCOTT (I966) observent un meilleur indice de consommation chez les poulettes Leghorn naines, et nous-mêmes (GUILLAUME, I969) notons que les sujets $d w$ stockent plus efficacement l'énergie mais utilisent moins bien les protéines de leur ration. Dans une expérience à caractère appliqué LECLERCQ, GUILLAUME et BLUM (I970) remarquent que des poulettes naines d'un croisement industriel ( $J V 15$, Selaf, France) contrairement aux poulets normaux (HILL et DANSKx, I954) n'augmentent pas leur consommation de nourriture quand le taux énergétique de leur ration est abaissé de 2900 à $2600 \mathrm{kcal} \mathrm{EM} / \mathrm{kg}$.

Les différentes observations laissaient supposer que le rapport calories/protéines $(\mathrm{C} / \mathrm{P})$ idéal pour des oiseaux normaux $(D w)$ pouvait ne pas 1'être pour des oiseaux nains $(d w)$. Nous avons donc effectué 3 essais successifs pour mettre en évidence une interaction entre génotype et équilibre énergie-protéines. Dans le I $^{\text {er }}$ essai nous avons fait appel à la technique dite "d'alimentation séparée " et laissé les animaux ajuster d'eux-mêmes leurs ingérés énergétique et protidique. Dans le $2^{\mathrm{e}}$ et le $3^{\mathrm{e}}$ essai, nous avons distribué des aliments complets de rapport calories/protéines variables selon un rationnement journalier strictement identique chez tous les animaux.

Dans les essais 2 et 3 nous avons en outre mesuré quelques constantes biochimiques ou physiologiques et la libération de radio-iode I3I afin de tenter d'éclaircir le mode d'action du gène.

\section{MÁTÉRIEL ET MÉTHODES}

Les animaux sont des poussins issus d'une souche expérimentale de RICARD (Station expérimentale d'Aviculture du Magneraud, I7 - France). Ils dérivent du croisement $D w d w \times d w$ - et comprennent par conséquent $50 \mathrm{p}$. Ioo de normaux et $50 \mathrm{p}$. roo de nains dans chaque sexe.

Tous sont placés en cages individuelles pendant de courtes périodes expérimentales : entre $2 \mathrm{r}$ et 28 jours dans le $\mathrm{I}^{\mathrm{er}}$ essai ; entre so et 24 jours dans le $2^{\mathrm{e}}$ et le $3^{\mathrm{e}}$. Ils sont ensuite élevés ensemble entre la fin de cet essai et la $12^{\mathrm{e}}$ semaine, âge auquel on effectue la détermination des phénotypes.

Lors de la répartition on regroupe les poussins en ensembles à l'intérieur desquels chaque traitement alimentaire est représenté deux fois a fin d'obtenir des " blocs » où tous les animaux sont d'une même famille et où se trouve le plus grand nombre possible de combinaisons génotype $\times$ traitement.

En fait l'analyse statistique est faite selon les cas soit par comparaisons de paires (søeurs 
différant par le génotype ou par le régime reçu) à l'aide du test $t$ de STUDENT soit, pour tester les effets globaux, par analyse de variance. Dans ce cas nous avons utilisé au mieux les dispositifs factoriels mis en œuvre dans le protocole, mais, du fait de l'inégalité des effectifs dans chaque groupe, il fallait éliminer au hasard un nombre important de données. Nous avons donc également effectué d'autres analyses de variance sans tenir compte des dispositifs factoriels.

\section{Expérience 1}

Elle porte sur 96 poussins parmi un lot de 150 animaux semi-pédigrés.

Le principe de l'alimentation séparée décrit par CALET et MELOT, I96I consiste à distribuer à chaque animal une ration d'un aliment riche en protéines pesée journellement et à lui laisser un aliment protéiprive à volonté. Dans cet essai, la ration protéique est augmentée au fur et à mesure de la croissance des poussins. Si elle est de $q$ dans le lot $\mathrm{r}$, elle est de I, $q q$ et I,7 $q$ (soit environ $\mathrm{I}, 3 \times \mathrm{I}, 3 q$ ) dans les lots 2 et 3 respectivement; le rapport de croissance des normaux à celui des nains est en effet voisin de I,3 (Guillaume, r969).

La composition des aliments est donnée dans le tableau $\mathrm{I}$.

\section{TABLEAU I}

Composition des aliments expérimentaux (en p. Ioo) de l'expérience 1

Expérience $\mathbf{I}$

\begin{tabular}{|c|c|c|}
\hline \multicolumn{3}{|c|}{ Aliment protéiprive } \\
\hline Glucose (cérélose) & $\ldots \ldots \ldots \ldots \ldots \ldots \ldots \ldots \ldots$ & 87,5 \\
\hline Huile de mais .... & 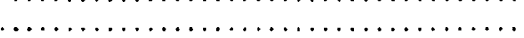 & 5 \\
\hline Cellulose. . . . . . . & 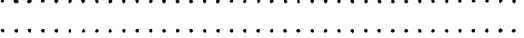 & 2 \\
\hline Choline à 25 p. 10 & 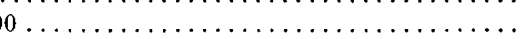 & 0,8 \\
\hline Mélange vitaminiqu & ${ }^{*} \ldots \ldots \ldots \ldots \ldots \ldots \ldots \ldots$ & 0,5 \\
\hline Mélange minéral ** & ${ }^{*} \ldots \ldots \ldots \ldots \ldots \ldots \ldots \ldots \ldots$ & 4,2 \\
\hline
\end{tabular}

Aliment protéiné

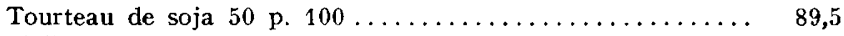

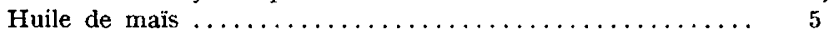

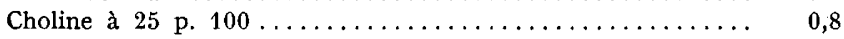

Mélange vitaminique ${ }^{*} \ldots \ldots \ldots \ldots \ldots \ldots \ldots \ldots \ldots \ldots \ldots \ldots, 0,5$

Mélange minéral $* * \ldots \ldots \ldots \ldots \ldots \ldots \ldots \ldots \ldots \ldots \ldots, 4,2$

* Apportant pour $100 \mathrm{~kg}$ : Vitamine A: $1600000 \mathrm{UI}$, Vitamine $\mathrm{D}_{3}$ : $100000 \mathrm{UI}$, Vitamine $\mathrm{E}: 10 \mathrm{~g}$, Vitamine $\mathrm{K}_{3}: 1 \mathrm{~g}$, Riboflavine : $3 \mathrm{~g}$, Panthoténate de $\mathrm{Ca}: 10 \mathrm{~g}$, Acide nicotinique : $25 \mathrm{~g}$, Thiamine : $2 \mathrm{~g}$, Pyridoxine : $3 \mathrm{~g}$, Biotine : $0,12 \mathrm{~g}$, Acide folique : $0,60 \mathrm{~g}$, Vitamine $\mathrm{B}_{12}: 0,01 \mathrm{~g}$, Acide ascorbique : $5 \mathrm{~g}$, Antioxydant : $10 \mathrm{~g}$, Amidon : q.s.p.

** Apportant en g pour $100 \mathrm{~kg}$ : Carbonate de Ca : 1500 , Phosphate bipotassique : 730, Chlorure de $\mathrm{Na}: 500$, sulfate de $\mathrm{Mg}, 7 \mathrm{H}_{2} \mathrm{O}: 500$, Phosphate bicalcique : 1700 , Sulfate de $\mathrm{Mn}, \mathrm{H}_{2} \mathrm{O}: 33$, Citrate ferrique :33, Alun de $\mathrm{K}$ : 1, Sélénite de $\mathrm{Na}: 0,055$, bromure de $\mathrm{Na}: 2,4$, Chlorure de $\mathrm{Zn}: 8$, Silicate de $\mathrm{Na}: 5,5$, Sulfate de $\mathrm{Co}, 7 \mathrm{H}_{2} \mathrm{O}: 0,2$, Molybdate de $\mathrm{Na}: 0,9$, Sulfate de $\mathrm{Cu}$, $5 \mathrm{H}_{2} \mathrm{O}: 2$, Acide borique : 0,9 , Iodure de $\mathrm{K}: 4$.

\section{Expérience 2}

Elle porte sur 96 poussins choisis parmi un lot de 217 animaux pédigrés. Tous reçoivent la même ration journalière qui passe de ro à $27 \mathrm{~g}$ du début à la fin de l'essai.

Les aliments dont la composition figure dans le tableau 2 sont de type maiss-soja et ont même niveau énergétique. L'un (régime $\mathrm{A}$ ) dose $20 \mathrm{p}$. Ioo de protéines et le second (B) 23,2 p. Ioo. La lysine et les acides aminés soufrés sont maintenus dans la même proportion par rapport à la pro- 
téine dans les 2 régimes; on peut donc dire que le régime $\mathrm{A}$ est suboptimal en protéines et acides aminés soufrés alors que le régime $B$ est nettement excédentaire par rapport aux besoins. Le rapport $\mathrm{C} / \mathrm{P}$ est de ${ }_{50}$ et $\mathrm{I} 30$ dans les régimes $\mathrm{A}$ et $\mathrm{B}$ respectivement.

\section{Expérience 3}

Il s'agit en quelque sorte d'une répétition de l'essai précédent sur des poussins issus des mêmes parents.

La seule variante porte sur l'alimentation : on utilise cette fois-ci le régime $\mathrm{A}$ et le régime $\mathrm{C}$ (tabl. 2) de taux énergétique plus élevé de ro p. roo environ $\left(C / P={ }_{5}{ }^{8}\right)$.

\section{TABLEAU 2}

Composition des régimes expérimentaux des expériences 2 et 3

\begin{tabular}{|c|c|c|c|}
\hline & \multicolumn{3}{|c|}{ Régimes } \\
\hline & A & B & $\mathrm{C}$ \\
\hline \multicolumn{4}{|l|}{ Composants } \\
\hline Tourteau de soja 50 p. $100 \ldots \ldots \ldots \ldots$ & 28,00 & 38,00 & 22,50 \\
\hline Farine de poisson 65 p. $100 \ldots \ldots \ldots \ldots$ & 0,00 & 0,00 & 4,00 \\
\hline Maïs $\ldots \ldots \ldots \ldots \ldots \ldots \ldots \ldots \ldots \ldots$ & 62,00 & 52,50 & 62,00 \\
\hline Huile d'arachide $\ldots \ldots \ldots \ldots \ldots \ldots \ldots$ & 2,50 & 3,00 & 5,85 \\
\hline Cellulose $\ldots \ldots \ldots \ldots \ldots \ldots \ldots \ldots$ & 0,85 & 0,00 & 0,00 \\
\hline Chlorure de choline à 25 p. $100 \ldots \ldots \ldots \ldots$ & 0,50 & 0,50 & 0,50 \\
\hline Complément vitaminique ${ }^{*} \ldots \ldots \ldots \ldots \ldots$ & 1,00 & 1,00 & 1,00 \\
\hline Phosphate bicalcique..$\ldots \ldots \ldots \ldots \ldots$ & 2,50 & 2,50 & 2,00 \\
\hline Carbonate de calcium $\ldots \ldots \ldots \ldots \ldots \ldots$ & 1,50 & 1,25 & 1,00 \\
\hline Sel iodé $\ldots \ldots \ldots \ldots \ldots \ldots \ldots \ldots$ & 0,50 & 0,50 & 0,50 \\
\hline Mélange oligo-minéral $* * \ldots \ldots \ldots \ldots \ldots$ & 0,50 & 0,50 & 0,50 \\
\hline DL méthionine $\ldots \ldots \ldots \ldots \ldots \ldots \ldots \ldots$ & 0,15 & 0,35 & 0,15 \\
\hline \multicolumn{4}{|l|}{ Analyse } \\
\hline Énergie métabolisable kcal/g (calculé) $\ldots . .$. & 3,01 & 3,00 & 3,29 \\
\hline$N \times 6,25$ (dosé) $\ldots \ldots \ldots \ldots \ldots \ldots \ldots$ & 20,0 & 23,2 & 20,9 \\
\hline 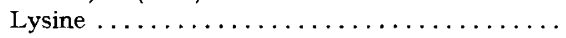 & 1,10 & 1,40 & 1,14 \\
\hline Acides aminés soufrés $\ldots \ldots \ldots \ldots \ldots \ldots \ldots$ & 0,80 & 1,00 & 0,82 \\
\hline 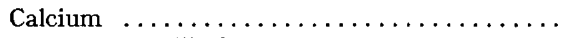 & 1,07 & 1,01 & 1,02 \\
\hline Phosphore utilisable ............... & 0,58 & 0,59 & 0,58 \\
\hline
\end{tabular}

* Apportant pour $100 \mathrm{~kg}$ : vitamine A : 730 000UI, vitamine $\mathrm{D}_{\mathbf{3}}$ : $93000 \mathrm{UI}$, vitamine $\mathrm{E}: 5000 \mathrm{UI}$, riboflavine : $0,4 \mathrm{~g}$, vitamine $\mathrm{B}_{12}: 0,093 \mathrm{mg}$, panthoténate de calcium : $0,53 \mathrm{~g}$, chlorure de choline : $100 \mathrm{~g}$, nicotinamide : $0,93 \mathrm{~g}, \mathrm{BHT}: 12 \mathrm{~g}$, avoine : q.s.p.

** Apportant en g pour $100 \mathrm{~kg}$ : sulfate de $\mathrm{Cu}, 5 \mathrm{H}_{2} \mathrm{O}: 1,05$, sulfate de Co, $7 \mathrm{H}_{2} \mathrm{O}: 0,15$, molybdate de $\mathrm{Na}: 0,075$, sulfate de $\mathrm{Zn}, 7 \mathrm{H}_{2} \mathrm{O}: 22,5$, iodure de $\mathrm{K}: 0,225$, carbonate de $\mathrm{Ca}: 50,25$, Fucus 7,5 , sulfate de $\mathrm{Mn}, 4 \mathrm{H}_{2} \mathrm{O}: 30$, oxyde de $\mathrm{Mg}, 7 \mathrm{H}_{2} \mathrm{O}: 15$, carbonate de $\mathrm{Mg}: 12$, sulfate de $\mathrm{Fe}, 7 \mathrm{H}_{2} \mathrm{O}: 11,25$.

\section{Dosages et mesures d'activité de la thyroide (exp. 2 et 3 )}

A l'âge de 12 semaines, les phénotypes étant déterminés, on choisit des paires de sœurs sur lesquelles on prélève du sang afin de doser le glucose sanguin (ro paires), les lipides totaux et le 
cholestérol ( 7 paires). Sur 4 paires de poulettes, on prélève également une fraction de $4 \mathrm{~g}$ de la masse musculaire pectorale afin de doser les acides aminés libres du muscle.

Le glucose sanguin est dosé à l'aide d'un autoanalyseur Technicon selon la méthode de HoFFMAN modifiée. Les lipides sanguins sont extraits à l'aide du mélange méthanol-chloroformeeau et le cholestérol est dosé sur des solutions chloroformiques de lipides à l'aide de l'autoanalyseur Technicon selon la méthode de Pearson, Stern et Mc Gavack, (I953) adaptée par Boy, Bonnafe et MAZET (I960). L'extraction et le dosage des acides aminés libres du muscle ont été faits selon les procédés décrits par LARBIER, GuILlaUme et CALET, (I97I) sur des poulettes à jeûn depuis I 5 h.

Pour estimer la sécrétion thyrö̈dienne, nous avons fait appel à la méthode de MELLEN et Wentworth, (1960). Io jours avant l'essai et jusqu'à la fin de celui-ci, les animaux choisis ont reçu un régime contenant $0, \mathbf{I}$. I oo de thiouracile afin d'empêcher la réutilisation de l'iode. A ce moment, on les pèse et on leur injecte $3 \mu \mathrm{Ci}$ d'iode $\mathrm{I}_{3} \mathrm{I}$ par roo g de poids vif. A partir du $2^{\mathrm{e}}$ jour après cet essai et tous les deux jours, on leur injecte de la thyroxine par voie intrapéritonéale selon la méthode que nous avons citée. Comme nous ignorions l'ordre de grandeur de la sécrétion thyroïdienne des poussins de ce type et de cet âge, la dose choisie assez arbitrairement varie de o, $5 \mu \mathrm{g}$ à $2,5 \mu \mathrm{g} / \mathrm{l}$ oo g de poids vif, du début à la fin de l'essai. La radioactivité retenue dans la thyroïde de l'animal est mesurée à l'aide d'un compteur sensible aux rayons gamma : Polyradiamètre DSM22 Nardeux, 37 - Loches (France), le récepteur étant appliqué directement sur l'animal au niveau de la glande.

Nous avons en outre dans un essai parallèle dosé les acides aminés libres du muscle, sur des poussins normaux d'origine commerciale âgés de 3 semaines et ayant reçu, soit un aliment usuel, soit le même régime enrichi de o,r p. roo de thiouracile durant ro jours.

\section{RÉSULTATS}

\section{Expérience 1}

Les résultats concernant la croissance, la consommation et l'efficacité alimentaire sont rassemblés dans le tableau 3 .

Chez les mâles des 2 génotypes, on retrouve un ajustement de l'ingestion des nutriments énergétiques à la ration azotée. Ce phénomène avait déjà été décrit : (Calet, Jouandet et Baratou, I96I ; Cale'T, Guillaume et Delpech, ig65). En revanche, cet ajustement n'est nullement évident chez les femelles où l'on observe au contraire, une légère diminution de la consommation d'éléments non protidiques quand la ration azotée passe de $6, \mathrm{I}_{5}$ à $8,0 \mathrm{~g} / \mathrm{j}$. Cette différence liée au sexe est rigoureusement la même avec les 2 génotypes. Indépendamment de la ration protéique, l'importance de la consommation d'éléments énergétiques et par suite le taux azoté de l'aliment total sont fonction du génotype : les individus nains mangent moins mais reconstituent une ration plus riche en protéines.

$\mathrm{Au}$ niveau de la croissance, on retrouve une répercussion du $\mathrm{I}^{\mathrm{er}}$ phénomène : pour les faibles rations azotées, les femelles qui ingèrent davantage d'aliment sans protéines croissent plus vite que les mâles ; la ration azotée de Io,4 $\mathrm{g}$ par jour rétablit 1a hiérarchie habituelle. Le choix d'un aliment plus riche en protéines par les nains se retrouvant dans les deux sexes, le rapport du poids des normaux à celui des nains est le même chez les mâles et les femelles : I,09 et $\mathrm{I}, 08$ respectivement. I1 semble que l'augmentation de la ration azotée tende à accentuer les différences entre nains et normaux chez les femelles, et à les diminuer chez les mâles. De toute façon, l'effet du sexe est plus important que celui du nanisme pour les faibies rations azotées (fig. I).

Des phénomènes dont nous venons de parler résultent de nettes différences entre nains et normaux au niveau de l'efficacité alimentaire : avec ce protocole expérimental, les poussins $d w$ utilisent les protéines moins efficacement que les normaux, 


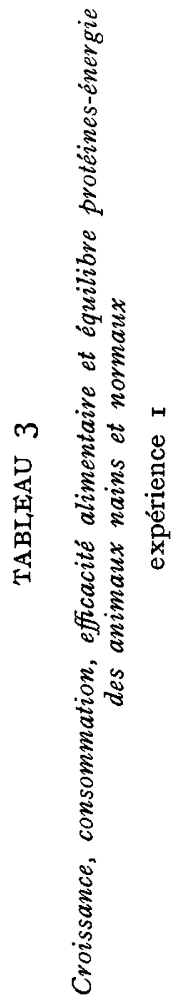

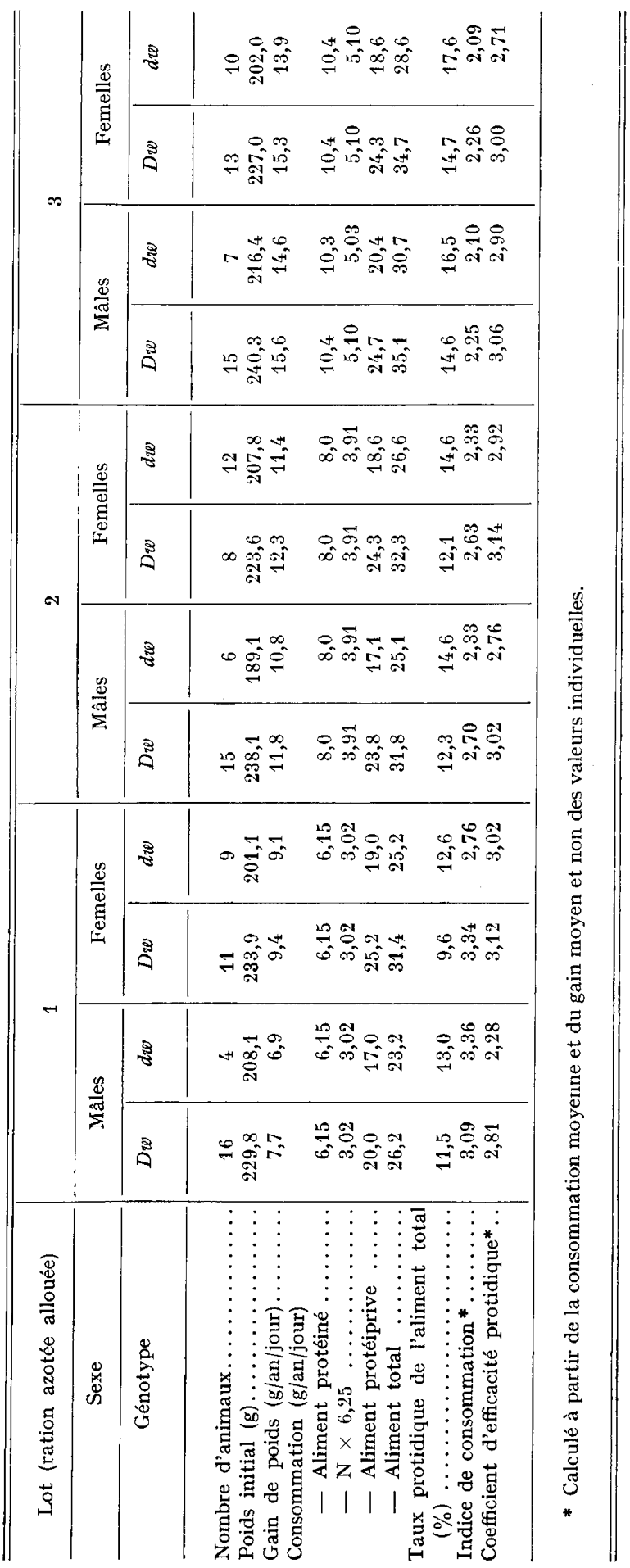


mais le taux de conversion de la ration totale est nettement en leur faveur. Une exception existe : le cas des mâles recevant la plus faible ration protidique, mais il est difficile d'y prêter beaucoup attention par suite du faible effectif des mâles nains de ce
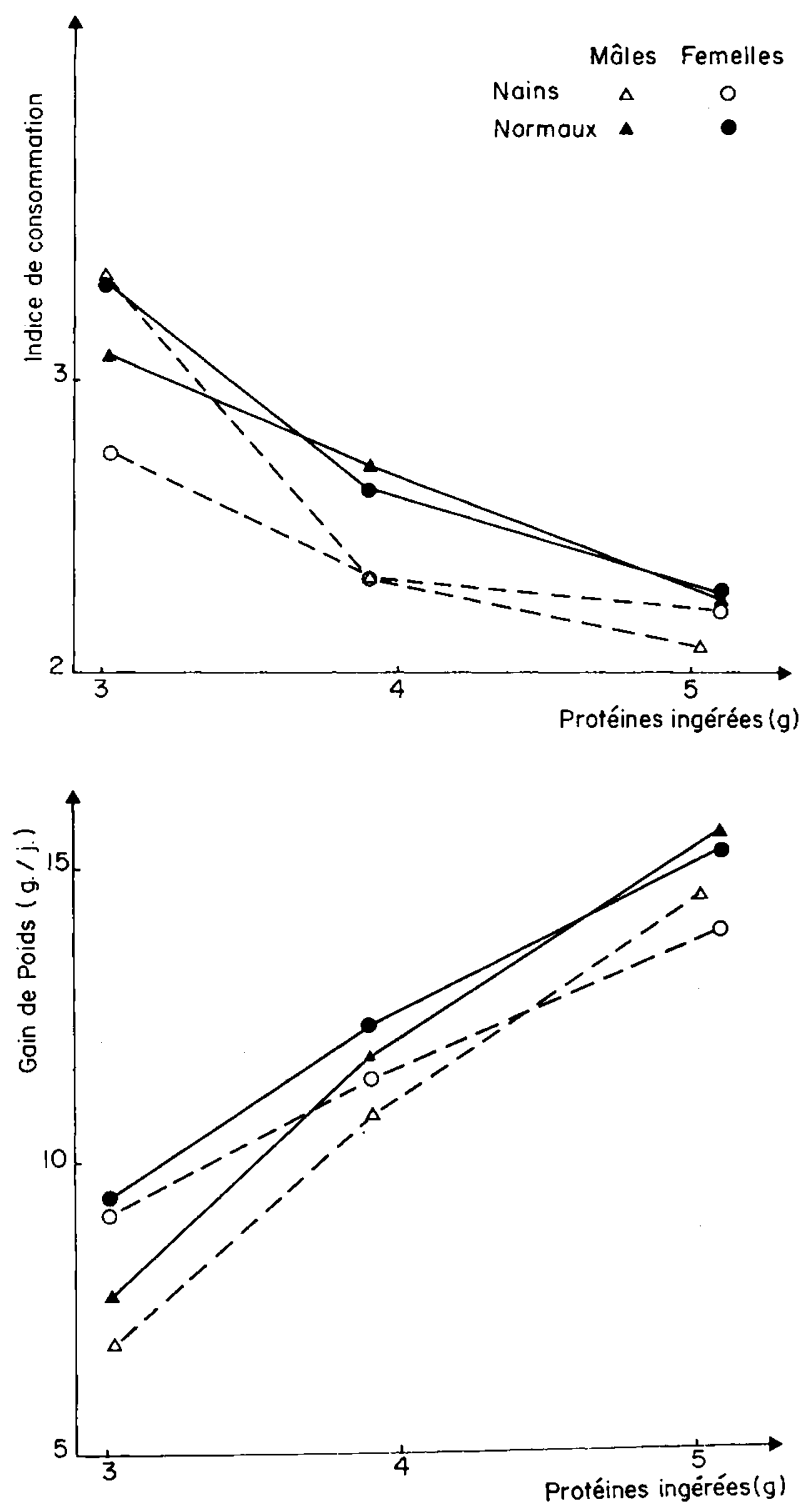

Frg. I. - Indice de consommation et gain de poids en fonction de la ration protéique allouée chez les poussins mâles et femelles, nains et normaux Expérience I

lot. A l'opposé, l'efficacité azotée mesurée par le coefficient d'efficacité protéique (CEP) est en faveur des sujets normaux dans tous les cas (fig. 2).

On pourrait objecter que, du fait de leur taille plus faible, les animaux nains 
recevaient des rations azotées relativement plus importantes que les normaux. Nous avons éliminé cette influence de la taille en étudiant les régressions de la consommation exprimée en p. Ioo du poids vif (taux d'ingestion) sur la croissance exprimée elle aussi en p. Ioo du poids vif (taux de croissance). Les résultats (tabl. 4) confirment bien une influence favorable du gène sur l'efficacité de la ration globale et défavorable sur celle des protéines.

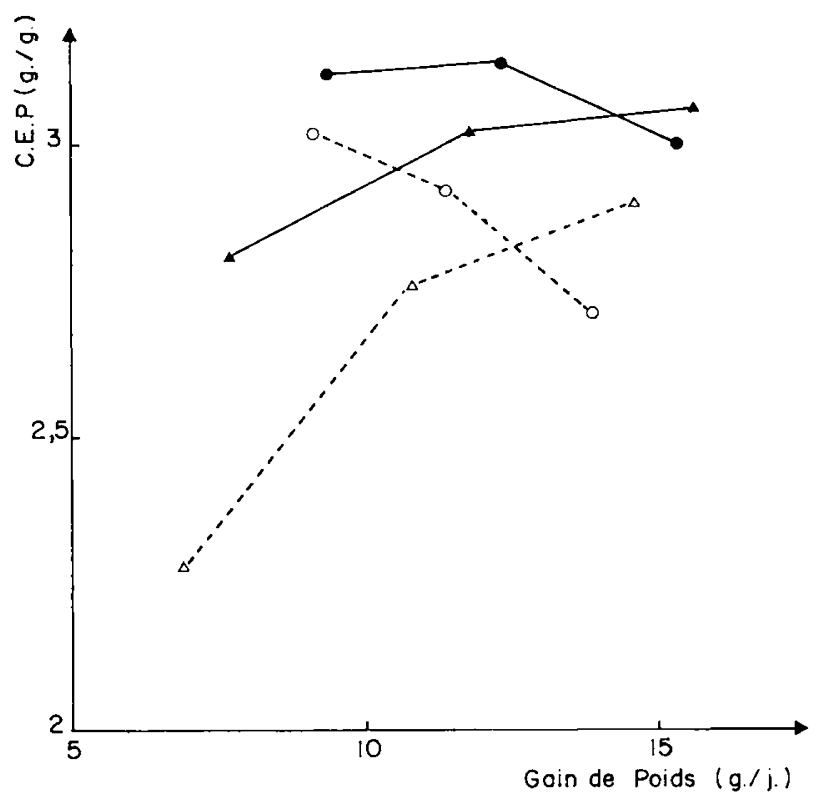

FIG. 2. - Variation du coefficient d'efficacité protéique en fonction du gain de poids chez les poussins mâles et femelles, nains et normaux

Expérience I : mêmes symboles que sur la figure I

\section{TABLEAU 4}

Relation entre taux d'ingestion $\left(y=\frac{\mathrm{I}}{\mathrm{P}}\right)$ et taux de croissance $\left(x=\frac{\Delta \mathrm{P}}{\mathrm{P}}\right)$

chez les poussins nains ou normaux

Expérience I

\begin{tabular}{|c|c|c|c|c|c|}
\hline & & $\begin{array}{l}\text { Équation } \\
\text { de régression }\end{array}$ & $r$ & $\begin{array}{c}\text { Nombre } \\
\text { de paires } \\
\text { de données }\end{array}$ & $\begin{array}{l}\text { Signification } \\
\text { de la régression }\end{array}$ \\
\hline $\begin{array}{l}\text { I : consommation } \\
\text { d'aliment total }\end{array}$ & $\begin{array}{l}D w \text {, mâles et femelles } \\
d w \text {, mâles et femelles }\end{array}$ & $\begin{array}{l}y=0,119 x+0,078 \\
y=0,083 x+0,084\end{array}$ & $\begin{array}{l}0,51 \\
0,60\end{array}$ & $\begin{array}{l}77 \\
47\end{array}$ & $\begin{array}{l}\mathrm{P}<0,01 \\
\mathrm{P}<0,01\end{array}$ \\
\hline $\begin{array}{l}\text { I : consommation } \\
\text { de protéines }\end{array}$ & $\begin{array}{l}D w, \text { mâles et femelles } \\
d w \text {, mâles et femelles }\end{array}$ & $\begin{array}{l}y=0,356 x+0,100 \\
y=0,394 x+0,108\end{array}$ & $\begin{array}{l}0,67 \\
0,67\end{array}$ & $\begin{array}{l}78 \\
47\end{array}$ & $\begin{array}{l}\mathrm{P}<0,01 \\
\mathrm{P}<0,01\end{array}$ \\
\hline
\end{tabular}




\section{Expériences 2 et 3}

Les résultats obtenus durant les périodes expérimentales des essais 2 et 3 sont consignés dans le tableau 5 .

Il est curieux de constater que dans l'expérience 3 , c'est-à-dire avec un rationnement sévère, une ingestion rigoureusement contrôlée d'aliment se traduit par le même gain de poids quels que soient le sexe et le génotype de l'animal. Dans ces conditions l'efficacité alimentaire globale n'est donc pas influencée par le génotype. Seule, la distribution du régime enrichi en protéines à la place du régime subcarencé modifie les performances, la croissance étant accrue de 5,3 p. Ioo. Cette amélioration globalement non significative apparaît cependant significative $(P<0,05)$ dans un cas :

\section{TABLEAU 5}

Gains de poids journaliers ( $\mathrm{g} / \mathrm{j})$ de poussins recevant des régimes enrichis en protéines (B) ou en énergie (C) Alimentation rationnée

Expériences 2 et 3

\begin{tabular}{|c|c|c|c|c|c|c|c|}
\hline & Phénotype & Sexe & A & B & $\mathrm{C}$ & $\begin{array}{c}\text { Moyenne } \\
\mathrm{A} \text { et } \mathrm{B}\end{array}$ & $\begin{array}{l}\text { Moyenne } \\
\text { A et C }\end{array}$ \\
\hline \multirow{4}{*}{$\begin{array}{c}\text { Expérience } 2 \\
\text { (consommation : } \\
18,2 \mathrm{~g} / \mathrm{j})\end{array}$} & \multirow{2}{*}{$D w$} & M & $8,9 \underset{(15)}{ \pm} 0,15$ & $9,2 \underset{(13)}{ \pm} 0,14$ & 一 & 9,1 & - \\
\hline & & $\mathbf{F}$ & $\underset{(14)}{8,8 \pm 0,15}$ & $9,6 \underset{(13)}{ \pm} 0,14$ & - & 9,2 & - \\
\hline & \multirow{2}{*}{$d w$} & M & $8,7 \underset{(9)}{ \pm} 0,14$ & $9,6 \underset{(8)}{ \pm} 0,14$ & - & 9,2 & 一 \\
\hline & & $\mathrm{F}$ & $8,8 \underset{(9)}{ \pm} 0,15$ & $9,1 \underset{(9)}{ \pm} 0,14$ & - & 9,0 & - \\
\hline \multirow{4}{*}{$\begin{array}{c}\text { Expérience } 3 \\
\text { (consommation : } \\
18,6 \mathrm{~g} / \mathrm{j} \text { ) }\end{array}$} & \multirow{2}{*}{$D w$} & M & $10,4 \underset{(15)}{ \pm} 0,14$ & - & $11,9 \underset{(8)}{ \pm} 0,25$ & - & 11,2 \\
\hline & & $\mathrm{F}$ & $\underset{(10)}{9,8 \pm 0,27}$ & - & ${ }_{(12)} \underbrace{}_{(12)} 0,26$ & - & 10,9 \\
\hline & \multirow{2}{*}{$d w$} & $\mathbf{M}$ & $8,8 \underset{(7)}{ \pm} 0,71$ & - & $10,9 \underset{(13)}{ \pm} 0,51$ & - & 9,9 \\
\hline & & F & $10,4 \underset{(12)}{ \pm} 0,18$ & - & ${ }_{(10)}^{ \pm 1,2} 0,28$ & - & 10,8 \\
\hline \multirow{2}{*}{ Expérience 2} & $\begin{array}{c}\text { Moyennes } \\
\text { sexes }\end{array}$ & $\int D w$ & 8,85 & 9,4 & - & 9,15 & - \\
\hline & confondus & $d w$ & 8,75 & 9,35 & - & 9,1 & - \\
\hline \multirow{2}{*}{ Expérience 3} & $\begin{array}{c}\text { Moyennes } \\
\text { sexes }\end{array}$ & $D w$ & 10,1 & - & 11,9 & 一 & 11,0 \\
\hline & confondus & $d w$ & 9,6 & 一 & 11,05 & - & 10,3 \\
\hline
\end{tabular}

Les nombres entre parenthèses représentent les effectifs. 
celui des femelles naines quand on compare entre elles les sœurs nourries de régimes différents. Sur l'ensemble des animaux, aucune interaction génotype $\times$ régime n'est significative.

Dans l'essai $n^{\circ} 3$, on distingue un certain effet du génotype : les poussins normaux consomment la même quantité d'aliment que les nains, croissent à une vitesse de 5,4 et $7 \mathrm{p}$. Ioo supérieure avec les aliments $A$ et $C$ respectivement. Dans ces mêmes conditions, l'effet du sexe est toujours inapparent. L'influence la plus marquée est, ici encore, celle de l'aliment $(\mathrm{P}<0,05)$ : L'élévation du taux énergétique améliore les performances de $\mathrm{I} 7,8$ et $\mathrm{I} 5,3 \mathrm{p}$. Ioo chez les normaux et les nains respectivement. Comme dans l'essai précédent, aucune interaction entre les facteurs étudiés ne s'avère significative. Néanmoins, une interaction du second ordre apparaît : les femelles normales tirent un meilleur parti de l'élévation du taux énergétique que les femelles naines $(\mathbf{P}<0,05)$.

\section{TABLEAU 6}

Teneurs du sang en lipides totaux, cholestérol et glucose de poussins nains ou normaux

\begin{tabular}{|c|c|c|c|}
\hline & $D w$ & $d w$ & $\begin{array}{l}\text { Signification } \\
\text { des résultats }\end{array}$ \\
\hline Lip. tot. ${ }^{*}(\mathrm{mg} / 100 \mathrm{ml})$ & $234,7 \pm 10,7$ & $210,3 \pm 22,6$ & NS \\
\hline $\begin{array}{l}\text { Cholestérol total } * \\
(\mathrm{mg} / 100 \mathrm{ml}) \ldots \ldots\end{array}$ & $57,0 \pm 3,3$ & $44,8 \pm 5,7$ & $p \simeq 0,05$ \\
\hline Cholest. ${ }^{*} \%$ des lipides & $2,43 \pm 0,11$ & $2,18 \pm 0,21$ & NS \\
\hline Glycémie $^{* *}$ (g/litre) & $1,64 \pm 0,04$ & $1,53 \pm 0,04$ & $p<0,05$ \\
\hline
\end{tabular}

\section{Caractéristiques biochimiques du sérum sanguin et du muscle}

Les résultats concernant la glycémie et la lipidémie sont rassemblés dans le tableau 6. On note que les sujets nains ont une glycémie significativement plus basse que les normaux; des différences de même sens existent également pour la lipémie et surtout pour le cholestérol total sanguin $(P<0,05)$ tandis que la teneur des lipides sériques en cholestérol n'est pas modifiée sensiblement.

Le dosage des acides aminés libres du muscle révèle une altération à la fois de la concentration de l'ensemble des acides aminés libres $(\mathrm{P}<0,05)$ et de leur équilibre (tab1. 7). Les acides aminés les plus diminués par le gène de nanisme sont, parmi les onze que nous avons dosés, la thréonine, la sérine et la glycine dont la concentration chute significativement; des baisses non significatives mais nettes sont également visibles par la valine et l'isoleucine. La diminution de l'ensemble des II acides aminés dosés atteint $32 \mathrm{p}$. Ioo. En comparaison, le thiouracile administré à des poussins normaux âgés de 3 semaines, dans l'essai parallèle mentionné plus haut, modifie à 
PAR'TICULARITÉS DU POUSSIN $d w$

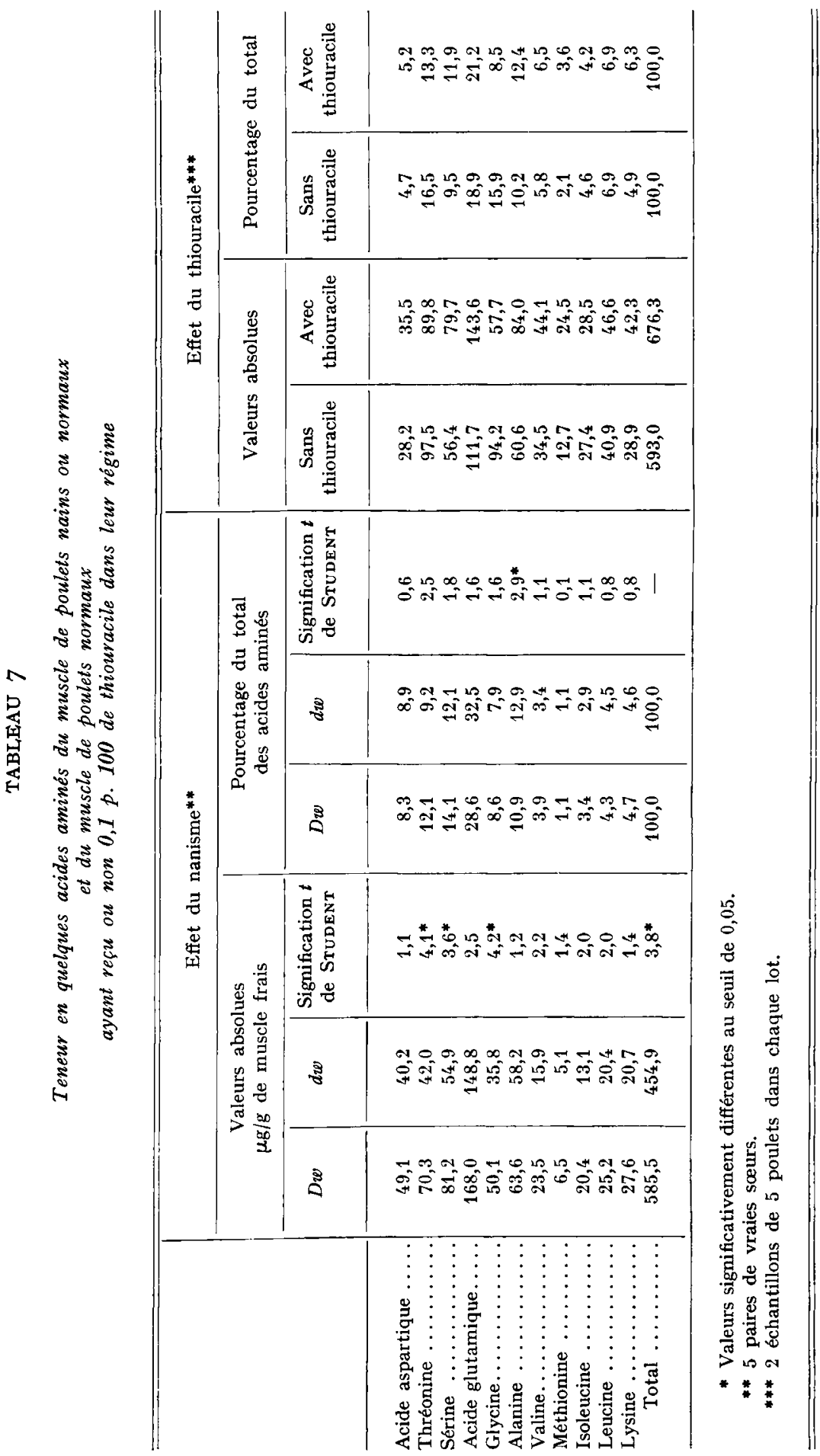


peine la concentration globale de ces II acides aminés. Il diminue également la concentration en glycine mais n'altère pas les teneurs en thréonine et isoleucine et augmente les quantités de sérine et valine.

TABLEAU 8

Effet de thiouracile sur la croissance de poussins nains ou normaux (animaux âgés de 12 semaines - Période de 10 jours)

Expérience 3

\begin{tabular}{|c|c|c|c|c|c|}
\hline & & \multicolumn{2}{|c|}{ Mâles } & \multicolumn{2}{|c|}{ Femelles } \\
\hline & & $D w$ & $d w$ & $D w$ & $d w$ \\
\hline $0 \%$ thiouracile & $\begin{array}{c}\text { Gain de poids } \\
(\mathrm{g} / \mathrm{j})\end{array}$ & $22,89 \underset{(13)}{ \pm} 1,87$ & $14,29 \underset{(7)}{ \pm} 0,75$ & $\underset{(10)}{13,65 \pm 1,21}$ & $8,14 \underset{(13)}{ \pm 0,86}$ \\
\hline $0,1 \%$ thiouracile & $\begin{array}{c}\text { Gain de poids } \\
(\mathrm{g} / \mathrm{j})\end{array}$ & $\underset{(12)}{21,54 \pm 1,73}$ & $11,43 \underset{(14)}{ \pm} 1,18$ & $\underset{(13)}{ \pm} 1,01$ & $3,70 \underset{(5)}{ \pm} 1,24$ \\
\hline
\end{tabular}

Les nombres entre parenthèses représentent les effectifs.

TABLEAU 9

Mobilisation de l'iode 131 chez les sujets nains ou normaux (Nombre de coups p. Ioo comptages précédents)

\begin{tabular}{|c|c|c|c|c|}
\hline \multirow{2}{*}{ Paire } & \multicolumn{2}{|c|}{$\begin{array}{l}\text { Rétention } \\
6 \mathrm{e} / 2^{\mathrm{e}} \text { jour }\end{array}$} & \multicolumn{2}{|c|}{$\begin{array}{l}\text { Rétention } \\
10^{\mathrm{e}} / 2^{\mathrm{e}} \text { jour }\end{array}$} \\
\hline & $D w$ & $d w$ & $D w$ & $d w$ \\
\hline 1 (søurs) $\ldots \ldots \ldots \ldots$ & 3 & 47 & 0 & 38 \\
\hline 2 (sœurs) ........... & 48 & 63 & 9 & 47 \\
\hline 3 (sœurs) .......... & 101 & 120 & 86 & 100 \\
\hline 4 (frères) .......... & 57 & 89 & 36 & 65 \\
\hline 5 (demi-sœurs) $\ldots \ldots$ & 56 & 60 & 50 & 48 \\
\hline 6 (sœurs) .......... & 5 & 6 & 0 & 3 \\
\hline 7 (demi-sœurs) . . . . & 40 & 101 & 36 & 92 \\
\hline 8 (frères) $\ldots \ldots \ldots \ldots$ & 48 & 25 & 37 & 41 \\
\hline Moyenne.......... & 44,75 & 63,87 . & 34,88 & 53,13 \\
\hline $\begin{array}{l}\text { Signification de la dif- } \\
\quad \text { férence } \ldots \ldots \ldots \ldots\end{array}$ & \multicolumn{2}{|c|}{$0,10<\mathrm{P}<0,05$} & \multicolumn{2}{|c|}{$\mathrm{P}<0,05$} \\
\hline
\end{tabular}

Nous avons aussi enregistré l'effet du thiouracile sur les animaux mâles et femelles, nains et normaux du lot destiné à la mesure de l'activité thyroïdienne (essai 3). Il apparaît clairement (tab1. 8) que le thiouracile a un effet dépressif sur la croissance 
de tous les animaux, sauf sur celle des femelles normales. Il ralentit significativement le développement des mâles nains et surtout celui des femelles naines. L'effet dépressif du thiouracile semble plus marqué chez les mâles nains que chez les mâles normaux mais l'interaction n'est pas significative. Comme cette drogue déprime davantage le développement des femelles naines que celui des femelles normales, il existe donc une interaction du $2^{e}$ ordre sexe $\times$ génotype $\times$ thiouracile. Il faut remarquer en outre que le thiouracile ne modifie jamais 1'homogénéité des résultats chez les normaux, mais multiplie le coefficient de variation chez les nains par 2 pour les mâles et par 3 pour les femelles.

Les résultats de l'estimation de l'activité thyroïdienne figurent dans le tableau 9. Il n'a pas été possible de chiffrer l'activité thyroïdienne dans toutes les paires, les doses de thyroxine injectées étant trop faibles dans la majorité des cas. Néanmoins, une différence nette apparaît entre nains et normaux : la rétention d'iode (retard de libération) est sytématiquement un peu plus forte chez les nains que les normaux ; il y a bien un effet de nanisme sur l'activité de cette glande, mais cet effet est faible en regard de la variation que l'on observe d'une famille à l'autre. Il n'est d'ailleurs significatif $(\mathrm{P}<0,05)$ qu'au Io jour après injection d'iode radioactif.

\section{DISCUSSION}

Pour interpréter l'adiposité accrue et l'utilisation moindre des protéines alimentaires, nous avions (GUILLAUME, I969) envisagé trois hypothèses relatives au mode d'action du gène $d w$ :

- Adiposité per se accrue.

- Hyperphagie et par suite obésité et gaspillage de protéines.

- Utilisation des protéines amoindries, d'où besoin d'une plus grande quantité de matières azotées, même pour synthétiser moins de protéines corporelles, d'où hyperphagie et obésité.

L'essai I permet de rejeter catégoriquement la seconde hypothèse : dans ce contexte nutritionnel les poussins nains consomment moins que les normaux par rapport à leur poids moyen et par rapport à leur besoin (Indice de consommation abaissé). La première hypothèse paraît, elle aussi, invraisemblable au vu des résultats de cet essai : on conçoit mal que les poussins nains aient été plus gras alors que leur efficacité alimentaire était meilleure.

Les résultats de l'essai I s'interprètent vraisemblablement par le $3^{\mathrm{e}}$ mécanisme envisagé : celui d'une réduction de l'utilisation des protéines. La distribution séparée des protéines et des éléments non azotés a permis aux poussins nains d'ingérer moins d'éléments énergétiques que les poussins normaux tout en consommant plus de protéines relativement à leur poids. L'efficacité protéique des premiers est plus mauvaise que celle des seconds quel que soit le mode d'étude choisi : gain de poids ou CEP en fonction de la dose de protéines ingérées (tabl. 3) gain de poids ou CEP en fonction du gain de poids (fig. 2). Dans cet essai, les poussins nains manifestent une meilleure utilisation alimentaire que les poussins normaux, ce qui n'est généralement pas observé. Ceci est surtout la conséquence du mode d'alimentation (alimentation séparée, 
qui permet au Poussin nain d'ingérer dans sa ration une proportion de protéines beaucoup plus grande que le poussin normal. En effet, pour un pourcentage de protéines donné le poussin nain a une moins bonne efficacité alimentaire que le normal (fig. 3). C'est donc grâce à une moindre consommation d'éléments non azotés, et à une surconsommation relative de protéines que le sujet $d w$ améliore son indice de consommation.

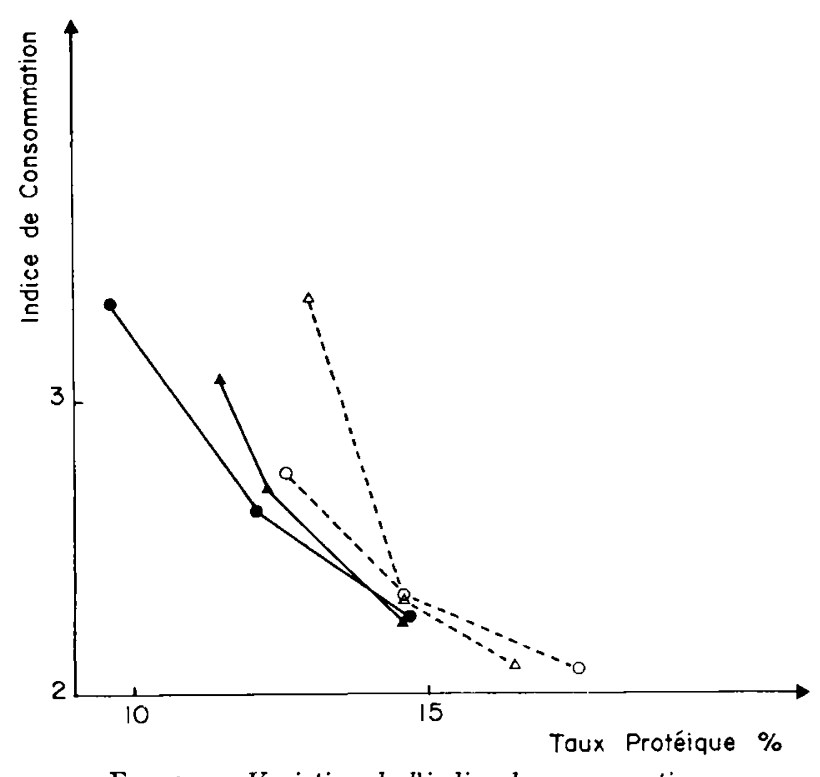

en fonction du taux protéique chez les poussins mâles et femelles, nains et normaux

Expérience I : mêmes symboles que sur la figure I

L'hypothèse d'un besoin azoté accru par le gène $d w$ est cependant - à première vue - peu compatible avec l'absence d'interaction entre taux azoté et nanisme, constatée en alimentation mixte et rationnée dans les essais 2 et 3 . Le désaccord entre 1'essai I d'une part et les essais 2 et 3 d'autre part ne peut provenir de la précision de l'essai (nombre d'animaux réduit mais comparable); il découle soit de l'âge des animaux, soit plus vraisemblablement du mode d'alimentation choisi. Le mode d'alimentation lui-même a pu influencer l'effet apparent du gène de plusieurs manières : par le rythme d'alimentation auquel les sujets nains réagissent différemment (Simon, I97I) ou par le rationnement quantitatif. Nous avons montré (GuILLAUME, I969) que les sujets nains utilisaient moins efficacement l'aliment pour la croissance mais plus efficacement pour l'entretien. Il est permis de penser que le rationnement choisi était voisin du niveau d'ingestion pour lequel ces deux effets s'annulent. Nous avions d'ailleurs vérifié dans l'expérience I que les faibles rations azotées minimisaient l'effet du gène de nanisme vis-à-vis de celui du sexe dans le choix d'un rapport $\mathbf{C} / \mathbf{P}$ (fig. I).

On ne peut, de toute façon, conclure à un effet défavorable du gène $d w$ sur 1'utilisation protéique du poussin en croissance dans tous les cas. Un rationnement sévère peut l'annuler. De même, l'effet défavorable de ce gène sur l'utilisation alim entair 
globale peut être supprimé ou même inversé par le mode d'alimentation. D'autres études sont donc nécessaires pour élucider l'effet du gène sur le métabolisme protéique et sur le besoin azoté.

Nos essais mettent en lumière l'importance du mode d'alimentation (et surtout du niveau d'ingestion) et démontrent l'existence d'un comportement alimentaire distinct chez les sujets nains et normaux en alimentation séparée. Ce dernier résultat est à rapprocher de ceux de LECLERCQ, GUILLAUME et BLUM (I970) en alimentation usuelle avec taux énergétiques variables et de ceux de Simon (I97I) en alimentation discontinue. Le gène $d w$ agirait donc sur la régulation de l'appétit. On ne peut dire s'il s'agit d'une action directe sur les centres nerveux ou d'une action indirecte par le biais des régulations métaboliques.

A l'appui de cette hypothèse, on peut rappeler les résultats de RAJARATnAm, SelivarajaH et Summers (I969) sur 1'abaissement de la température corporelle chez les sujets $d w$. On sait en effet que la température corporelle, sans être un reflet sûr de 1'hypothyroïdisme (D'Angelo, I963; Pastro, March et Biely, I969 $a$ et $b$ ) a été considérée comme un élément de régulation de l'appétit (BROBEK, I960). Il en est de même de la glycémie (MAYER, I957). L'abaissement de la glycémie constaté ici, fournit un autre argument en faveur d'une perturbation de l'appétit par le biais de modifications du métabolisme.

L'origine de ces modifications, c'est-à-dire en définitive le mode d'action $\mathrm{I}^{\mathrm{er}} \mathrm{du}$ gène n'a pas encore été démontrée. Les travaux de RAJARATNAM, SELVARAJAH et SuMMERS (I969) et de MÉRAT et Guillaume (Ig69) laissaient supposer l'existence d'un hypothyroïdisme. Nos résultats obtenus ici, fondés sur des tests plus sûrs, permettent de la confirmer mais aussi de limiter l'importance qu'il convient de lui attribuer. Puisque les différences d'activité thyroïdienne d'une famille à l'autre dépassent celles qui existent entre frères et sœurs, l'un nain l'autre normal, l'hypothyroïdisme ne saurait à lui seul être la cause du nanisme. Cette conclusion est d'ailleurs en accord avec celles de Van Tienhoven et al. (ig66), Rajaratnam, Selvarajah et Summers (I969), Guirlaume (I970).

Les constantes sanguines que nous avons mesurées ne fournissent guère d'indications précises : l'abaissement de la glycémie peut être la conséquence de l'équilibre de nombreuses hormones (glucagon, insuline, glucocorticostéroïdes, thyroxine, hormones de croissance). La diminution de la cholestérolémie serait chez un mammifère contraire aussi bien à un hypothyroïdisme - pourtant confirmé chez nos animaux (JURAND et OlIVER, 1970) qu'à une déficience en hormones de croissance (BUYERS, FrIEDMAN et Rosenman, I970). I1 faut donc dans ce cas précis admettre 1'existence d'un autre déséquilibre endocrinien à effet majeur ou bien d'une réponse différente des mammifères et des oiseaux.

Les variations de concentration et d'équilibre des acides aminés libres des tissus ont des causes multiples et encore mal connues (ANONYME, I969). Néanmoins parmi les modifications d'origine hormonale signalées dans la littérature, seule celle qui correspond à la déficience d'hormone somatotrope (ZACHMAN, I969) est comparable à celle que nous avons observée. Certes cet auteur a travaillé sur une espèce très éloignée (Homme) et un tissu tout différent (sang). Néanmoins, nous observons la même diminution globale des acides aminés libres et surtout la même altération de leur équilibre (à l'exception de la méthionine dont la concentration trop faible dans le muscle ne peut donner lieu à aucune conclusion). Ces changements sont très diffé- 
rents de ceux qui résultent d'un hypothyroïdisme provoqué par le thiouracile. Nous pensons donc, que sans constituer un argument décisif, la modification des acides aminés libres du muscle renforce la vraisemblance d'une relation entre gène $d w$ et hormone de croissance. Cette hypothèse reste cependant à démontrer. Dans la mesure où elle serait vérifiée, rien ne permet encore de dire si cette relation est due à la sécrétion ou la libération d'hormone de croissance ou à la sensibilité des tissus.

\section{CONCLUSION}

De l'ensemble de ces 3 essais, il est difficile de conclure que le poussin nain nécessite un régime à rapport Calories/Protéines moins élevé que le normal. Les résultats apparemment nets de la première expérience peuvent en effet signifier modification du comportement alimentaire aussi bien qu'élévation du besoin azoté. Les résultats des expériences 2 et 3 , bien que peu marqués, sont cependant dans le sens d'un besoin azoté accru par le nanisme chez les femelles. Il est permis de penser que la dominance incomplète de l'allèle $D w$ a masqué chez les mâles ( $D w d w$ et $d w d w)$ ce phénomène, par ailleurs atténué par le rationnement. Nous envisageons donc de poursuivre l'étude de l'influence du gène $d w$ sur le besoin azoté dans d'autres conditions nutritionnelles.

Sur un plan plus théorique, les observations biochimiques et physiologiques faites dans ces essais nous incitent à rechercher si le gène $d w$ modifie la sécrétion ou la libération d'hormone de croissance ou la sensibilité des tissus à cette hormone.

Reçu pour publication en octobre 1971.

\section{SUMMARY}

\section{SOME NUTRITIONAI, AND PHYSIOLOGICAI, TRAITS OF THE DWARF $d w$ CHICK}

Very little information is available concerning the nutrient requirement of the growing $d w$ chick as compared to the normal chick. Since we found in a previous experiment that the $d w$ chick used energy more efficiently and protein less efficiency we attempted to determine whether or not there existed an interaction between protein level and genotype in three different short duration experiments. All the birds were either pedigreed or semi-pedigreed chicks and resulted from the cross $D w d w \times d w$ - Therefore we had 50 per cent normal and 50 per cent dwarf chicks of each sex. Phenotypes were determined after the end of the trials at 12 weeks of age.

In Trial I all the bird were fed with the method of "separate feeding ". They received a daily limited amount of a protein-rich diet and in an another feeder a protein free diet ad libitum Three different amounts of protein-rich diet were chosen. Under these conditions the proteinfree diet intake of the females was not related to the amount of protein-rich diet alloted. In contrast, males made a balance between the protein-free and protein-rich diets, that it to say between calories and protein. In both sexes the protein level in terms of percentage of total intake was higher with dwarf birds.

As a result their feed efficiency was higher but their protein efficiency ratio was lower.

In Trial 2 all the birds were limit-fed the same amount of feed, Two isocaloric diets were tested : diet A, marginal in protein and diet B containing an excess of protein.

There were only slight differences in growth between groups. and no general interaction of protein level $\times$ genotype could be demonstrated. However, with pair comparisons it was shown that increasing the protein level significantly improved weight gain only with dwarf females.

In Trial 3 the same experimental procedure was followed but instead of diet $B$ we used a diet of the same protein content as diet A but with a higher energy level (diet C). Differences of 
weight gain due to genotype or diet were more pronounced. There was again no general interaction between genotype and diet. However, there was a significant interaction $(p<0.05)$ between genotype and energy level in the females, growth being increased to a greater extent in the normal than in the dwarf pullets.

In conclusion it seems probable that for maximal growth growing dwarf birds need diets with a higher protein level or lower calorie to protein ratio. The phenomenon seems especially true for females. In males the lack of differences due to diets may be due to the fact that "normal" birds were heterozygotes. All differences in feed efficiency were reduced with limited feeding, the results were influenced by the feeding level. Some differences in feeding behaviour between $d w$ and $D w$ may also play a role.

At 12 weeks of age a few biochemical and physiological measurements were also made in pairs of sisters or brothers (one $d w$ and one $D w$ ). The $d w$ birds differed markedly from $D w$ by their lower blood levels of sugar, total lipids and cholesterol. The free amino acid contents of the muscle was lower and the amino acid pattern was also modified in the dwarf pullets. Threonine, serine and glycine were significantly reduced. The concentrations of valine and isoleucine were also strongly though not significantly reduced. These modifications are very different from those produced by feeding thiouracil.

Feeding o. Io per cent thiouracil at 12 weeks of age during ten days had a slight non-significant growth depressing effect in males. In females it significantly reduced growth only in $d w$. Measurement of the release of 131 I by the thyroid did not provide an estimation of the thyroid hormones secretion rate but a significant difference between $d w$ and $D w$ was demonstrated. However, the differences between $d w$ and $D w$ sisters within families were small compared to differences between families.

It is suggested that the decreased activity of the thyroid is a secondary effect of the gene and is not responsible for the dwarfism effect. The latter would be due to a decrease of growth hormone secretion. or to a decrease of susceptibility of tissues to this hormone.

\section{RÉFÉRENCES BIBLIOGRAPHIQUES}

Anonyme, I969. Factors causing changes in plasma amino acid patterns. Nutr. Rev.,27, $24 \mathrm{I}-244$.

Bernier P. E., Arscott G., 1966. Growth and feed requirements of dwarf White Leghorn pullets as compared to their normal size sisters. Poult. Sci., 45, ro7o (Abstr.).

Boy J., Bonnafe M., Mazet J. B., I960. Determination du cholestérol total par une modification de la méthode de Pearson. Son emploi par voie manuelle et automatique. Ann. Biol. Clin., Paris, 18, 669-675.

Brobeк J. A., I96o. Hormones and metabolism : Food and temperature. Recent Progr. Hormone Res., 16, 439-459.

Buyers S. O., Friedman M., Rosenman R. M., r97o. Prevention of hypercholesterolaemia in thyroïdectomized rats by growth hormone. Nature, 228, 464-465.

Calet C., Jouhandet C., Baratou J., I96I. Variations de la consommation spontanée d'énergie du poussin en fonction de la nature des matières azotées du régime. Ann. Biol. anim. Bioch. Biophys., 1, 5-9.

Calet C., Melot M., I96I. Efficacité comparée pour la croissance du poussin de la méthionine et du sel de calcium de l'acide hydroxyméthylthiobutyrique. Ann. Zootech., r961, 10, 205-213.

Calet C., Guillaume J., Delpech P., I966. Le rôle des protides dans l'utilisation des nutriments énergétiques chez le Poussin. Amino acides, Peptides, Protéines. Cahiers de l'A.E. C., n⿳0 6, 265-282.

D'ANgelo S. A., I963. Central nervous regulation of the secretion and release of thyroïd stimulating hormone in Advances in Neuroendocrinology, ed. : A. V. Nalbandov University of Illinois Press. Urbana Ill., I86-I87.

Guillaume J., rg69. Conséquences de l'introduction du gène de nanisme $d w$ sur l'utilisation alimentaire chez le Poussin femelle. Ann. Biol. anim. Bioch. Biophys., 8, 369-378.

Guillaume J., 1970. Étude comparée du métabolisme de la Poule naine $d w$ et de la Poule normale. A. Ve Symposium energy metabolism of farms animals, Zurich, sept. I970. E. A. A. P. publication $n^{\circ} 13$, 217-2I9 (Abstr.).

Hill F. W., Dansky L. M., I954. Studies on the energy requirements of chickens. I. The effect of dietary energy level on growth and feed consumption. Poult. Sci., 33, I I2-I I9.

Hutr F. B., r953. Sex linked dwarfism in the fowl. Genetics, 28, 670.

Hutt F. B., I959. Sex linked dwarfism in the fowl. J. Heredity, 40, 209-221.

JaAP R. G., Монамmadian M., I969. Sex linked dwarfism and egg production of broiler dams. Poult. Sci., 48, 344-346.

Annales de génétique animale. - I972. 
JuRAND J., Oliver M. F., I970. Effect of thyroid activity on fatty acid composition of serum lipids. Altherosclerosis, 11, I25-140.

Larbier M., Guillaume J., Calet C., I97I. Le maïs grain : traitement de séchage et qualité. VII. Disponibilité de la lysine chez le poulet. Ann. Zootech., 20, $\mathrm{n}^{\circ} 45$ (sous presse).

Leclerce B., Guillaume J., Blum J.-C., 1970. Données sur les besoins alimentaires de la reproductrice naine Vedette I. N. R. A. (dw) durant les périodes de croissance et de ponte. I. Période de croissance. Communication XIV Congrès Mondial d'Aviculture, Madrid, sept. I97o (sous presse).

MAYer J., 1956. La régulation de l'appétit. $X X^{\mathrm{e}}$ Congrès International de Physiologie, Bruxelles, 138 -158.

Mellen W. J., Wentwortm B. C., I96o. Comparison of methods for estimating thyroïd secretion rate in chickens. Poult. Sci., 39, 678-686.

Mérat P., I969. Étude d'un gène de nanisme lié au sexe chez la Poule. I. Description sommaire et performances. Ann. Génét. Sélect. anim., 1, 19-20.

Mérat P., Gulllaume J., ig69. Étude d'un gène de nanisme lié au sexe chez la Poule. II. Fonctionnement thyroïdien. Ann. Génét. Sél. anim., 1, I31-133.

Mohammadian M., I969. Physiological effects of the sex linked dwarfing gene dw in broilers. Poult. Sci., 48, I845-1846 (Abstr.).

Pastro K. R., March B. E., Biely J., I969a. Body temperature of chicks in response to lysine deficiency. Can. J. Physiol. Pharmacol., 74, 339-342.

Pastro K. R., March B. E., Biely J., $x 969 b$. Diminished thyroidal activity in chicks in response to lysine deficiency. Can. J. Physiol, Pharmacol., 74, 645-647.

Pearson S., Stern S., McGavack T. H., I953. A rapid, accurate method for the determination of total cholesterol in serum. Ann. Chem., 25, 813-814.

Rajaratnam G., Selvarajah T., Summers J. D., I969. The effect of thyroprotein on growth rate of dwarf pullets. Poult. Sci., 48, I768-1772.

Ricard F. H., I97o. Étude d'un gène de nanisme lié au sexe chez la Poule. V. Observations sur la croissance et les caractéristiques de carcasse du jeune poulet. Ann. Génét. Sélect. anim., 2, 19-3I.

Simon J., I97I. Influence du gène de nanisme $(d w)$, du gène cou nu ( $\mathrm{Na}$ ) et du rythme d'alimentation sur la croissance et le comportement alimentaire du Poulet. Ann. Génét. Sélect. anim. 4.

Van Tienhoven A., Williamson J. H., Tomlinson M. C., Mcinnes K. L., ig66. Possible role of the thyroïd and the pituitary glands in sex linked dwarfism in the fowl. Endocr., 78, 905-957.

ZACHMAN M., 1969. Influence of human growth hormone (H. G. H.) on plasma and urine amino acid concentrations in hypopituitary dwarfs. Acta Endocrinol., 62, 513-520. 